SCIENCE CHINA

Physics, Mechanics \& Astronomy

\title{
Geometrical characterization of reduced density matrices reveals quantum phase transitions in many-body systems
}

\author{
ZhenSheng Yuan* \\ Department of Modern Physics, University of Science and Technology of China, Hefei 230026, China
}

Received February 21, 2017; accepted February 24, 2017; published online April 20, 2017

Citation: Z. S. Yuan, Geometrical characterization of reduced density matrices reveals quantum phase transitions in many-body systems, Sci. China-Phys. Mech. Astron. 60, 060331 (2017), doi: 10.1007/s11433-017-9014-8

Quantum phase transitions (QPTs) play a central role for understanding many-body physics [1]. Different from classical phase transitions which are driven by thermal fluctuations, QPTs are driven by quantum fluctuations at zero temperature and can be accessed by varying some physical parameters of the many-body system. Characterizing QPTs, which normally needs complicated theoretical calculations, becomes a fundamental problem to further study quantum matters. Here a group of physicists proposed to connect the geometrical properties of reduced density matrices (RDMs) of the physical system with its quantum phase transitions $[2,3]$.

For a many-body system (with $N$ particles) described by a Hamiltonian $H(\lambda)$ containing some set of parameters $\lambda$, the ground state $\left|\psi_{0}^{N}(\lambda)\right\rangle$ may change suddenly while the parameter $\lambda$ changes smoothly, leading to a QPT. To make QPTs more visible, instead of analyzing $\left|\psi_{0}^{N}(\lambda)\right\rangle$ itself, the authors proposed to characterize geometrically the two-particle reduced density matrices (2-RDMs) of $\left|\psi_{0}^{N}(\lambda)\right\rangle$, which would reflect the sudden change of the ground state $[2,3]$.

Concretely, the RDMs of many-body quantum states form a convex set. The boundary of low dimensional projections of this convex set may exhibit nontrivial geometry such as ruled surfaces. For example, the three-dimensional projection $\Theta$ is convex in $\mathbb{R}^{3}$. Ruled surfaces emerges from the boundary $\partial \Theta$ of $\Theta$. Shown in Figure 1 are the ruled surfaces for the Ising model in the large $N$ limit.

The authors studied the physical origin of these ruled sur-

*Correspondence author (email: yuanzs@ustc.edu.cn)

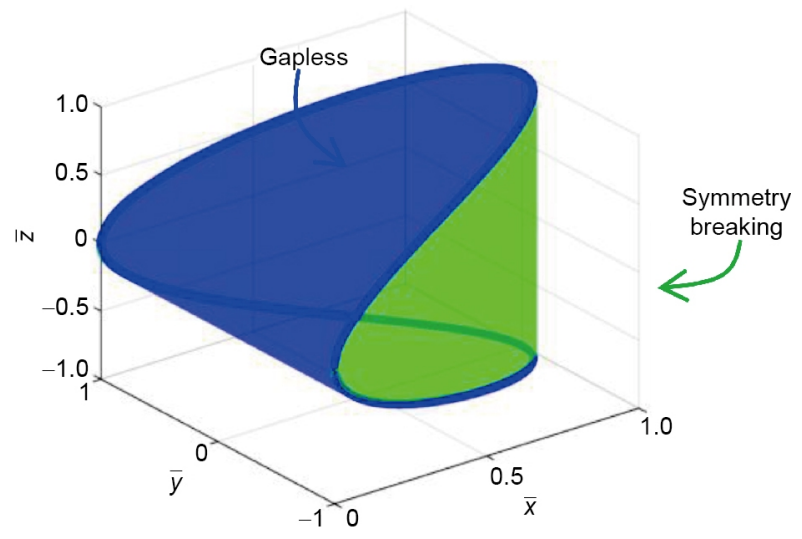

Figure 1 (Color online) Convex set for Ising model in the large $N$ limit. It is determined by $\bar{x}=\bar{z}^{2}$ for $\bar{y}^{2}+\bar{z}^{2} \leq 1$ and $\bar{x}+\bar{y}^{2}=1$ for $\bar{y}^{2}+\bar{z}^{2} \leq 1$. Here, $\bar{x}=\operatorname{tr}\left(\rho_{2}^{\infty}(X \otimes X)\right), \bar{y}=\operatorname{tr}\left(\rho_{2}^{\infty}(Z \otimes I)\right)$ and $\bar{z}=\operatorname{tr}\left(\rho_{2}^{\infty}(X \otimes I)\right)$. The green ruled surface is due to symmetry breaking, while the blue ruled surface is due to the fact that the system is gapless in that region.

faces for bosonic systems. The emergence of ruled surfaces was recently proposed as signatures of symmetry-breaking phase [4]. Apart from this, ruled surfaces can also be the consequence of gapless quantum systems [2]. By the quantum de Finetti's theorem [5], in the limit of large system size, these RDMs are the convex set of all the symmetric separable states. To distinguish ruled surfaces originated from gapless systems from those caused by symmetry-breaking, the authors propose to use the finite size scaling method for the corresponding geometry. This method is then applied to the two-mode XY model, successfully identifying a ruled surface as the consequence of gapless systems [2]. 
Moreover, the authors found the physical connections between the ruled surfaces and the joint product numerical range $\Pi$ of the Hamiltonian interaction terms. They verified that a ruled surface on $\partial \Theta$ sitting in $\Pi$ has a gapless origin, otherwise it has a symmetry-breaking origin [3].

Based on these results [2,3], the geometrical properties of the RDMs directly reflect the QPTs of quantum many-body systems. This offers a new perspective for understanding quantum phase transitions. One may envision that these theories will contribute to the research of quantum simulation $[6,7]$ with quantum many-body systems, say with ions [8], ultracold atoms [9], or photons [10] and will gradually attract more attentions in the related research fields.

1 S. Sachdev, Quantum Phase Transitions (Cambridge University
Press, Cambridge, 2011).

2 J. Y. Chen, Z. Ji, Z. X. Liu, X. Qi, N. Yu, B. Zeng, and D. Zhou, Sci. China-Phys. Mech. Astron. 60, 020311 (2017).

3 J. Chen, C. Guo, Z. Ji, Y. T. Poon, N. Yu, B. Zeng, and J. Zhou, Sci. China-Phys. Mech. Astron. 60, 020312 (2017).

4 V. Zauner, D. Draxler, L. Vanderstraeten, J. Haegeman, and F. Verstraete, New J. Phys. 18, 113033 (2016).

5 E. Størmer, J. Funct. Anal. 3, 48 (1969).

6 R. P. Feynman, Int. J. Theor. Phys. 21, 467 (1982)

7 A. Trabesinger, Nat. Phys. 8, 263 (2012).

8 D. Leibfried, R. Blatt, C. Monroe, and D. Wineland, Rev. Mod. Phys. 75, 281 (2003).

9 I. Bloch, J. Dalibard, and W. Zwerger, Rev. Mod. Phys. 80, 885 (2008), arXiv: 0704.3011.

10 J. W. Pan, Z. B. Chen, C. Y. Lu, H. Weinfurter, A. Zeilinger, and M. Żukowski, Rev. Mod. Phys. 84, 777 (2012), arXiv: 0805.2853. 